\title{
ORIGINAL
}

\section{VIOLENCIA DE LOS USUARIOS HACIA EL PERSONAL DE ENFERMERÍA EN LOS HOSPITALES PÚBLICOS DE LA REGIÓN DE MURCIA (*)}

\author{
Inmaculada Galián Muñoz (1), Bartolomé Llor Esteban (2) y José Antonio Ruiz Hernández (3).
}

(1) Servicio de Prevención de Riesgos Laborales. Servicio Murciano de Salud. Murcia. España.

(2) Facultad de Enfermería. Universidad de Murcia. Murcia. España.

(3) Facultad de Psicología. Universidad de Murcia. Murcia. España

(*) Este trabajo forma parte de un proyecto de investigación más amplio sobre violencia laboral en personal de enfermería, financiado por el Ministerio de Trabajo y Asuntos Sociales, Secretaría General de Políticas de Igualdad, Instituto de la Mujer (Expediente 152/07).

\section{RESUMEN}

Fundamentos: El riesgo de violencia laboral presenta una especia importancia en el ámbito sanitario, siendo el colectivo de enfermería uno de los más afectados. El objetivo es conocer la prevalencia de manifestaciones hostiles de los usuarios hacia los profesionales de enfermería hospitalaria dependientes del Servicio Murciano de Salud (SMS) y detectar aquellas características sociodemográficas y laborales de los trabajadores asociadas a una mayor exposición.

Métodos: Estudio descriptivo transversal llevado acabo en 2010 en el personal de enfermería de todos los hospitales del SMS a través de una encuesta autoaplicada y anónima que contenía la escala ECOH-U. El muestreo fue aleatorio y estratificado por hospitales y servicios $(30 \% \mathrm{de}$ los trabajadores), obteniendo finalmente un tamaño muestral de 1.489 sujetos (nivel de confianza 99\%; error muestral 1,75\%). Se comparó la media de puntuación obtenida en la escala según variables sociodemográficas y laborales, utilizando la prueba t de Student en variables dicotómicas y ANOVA y Tukey en variables multirrespuesta.

Resultados: El 21,8\% de las personas encuestadas refirió haber sufrido "Enfados por la demora asistencial" con una frecuencia al menos mensual. Los trabajadores del hospital psiquiátrico $(19,7)$, los de urgencias $(20,60)$, los que tienen contratos temporales $(16,38)$ y una antigüedad 6-10 años $(17,20)$ obtuvieron puntuaciones significativamente mayores en la escala.

Conclusiones: La distribución del riesgo no es homogénea dentro del colectivo. Se han hallado diferencias significativas en las agresione recibidas según estado civil, edad, hospital, servicio, profesión, tipo de contrato, turno y antigüedad en la profesión

Palabras clave: Agresion. Escala. Violencia laboral. Salud laboral. Servicio de enfermeria en hospital.

Correspondencia

Inmaculada Galián Muñoz

Barcelona, 57

Las Torres de Cotillas

30565 Murcia

Correo electrónico: inmaculada.galian@carm.es

\section{ABSTRACT}

\section{User Violence towards Nursing Staff in Public Hospitals. Murcia, Spain}

Background: The workplace violence has special relevance for the health care workers. Nursing staff is one of the professions most affected by this risk. Our objective is to determine the prevalence during the past year of diverse hostile manifestations by users towards professional hospital nursing staff who depend on the "Servicio Murciano de Salud" [Health Service of Murcia] (SMS), as well as to detect the sociodemographic and occupational workers characteristics associated with higher exposure.

Methods: A cross-sectional study carried out during the year 2010 of a random sample of nursing personnel from all the hospitals of SMS, through a self-administered and anonymous survey (Ecoh-U scale). The sample was stratified by hospitals and services (30\% of the workers) and finally we got a sample of 1.489 workers (confidence level $99 \%$; sampling error $1,75 \%)$. We compared the punctuation average obtained in the scale according to variables sociodemographics and laborables. We used the test $t$ of student in variables dichotomous and ANOVA and Tukey in variables multi-response.

Results: The $21,8 \%$ of the surveyed people reported that they suffered from "anger due to assistential delay" at least once a month. The workers who obtained punctuations significantly larger were psychiatric hospital workers $(19,7)$, emergency workers $(20,60)$, temporary $(16,38)$ and with old 6-10 years in the profession $(17,20)$.

Conclusions: Although nursing staff is one of the professions most exposed to violence, the risk distribution is not homogeneous. Significant differences were found according to marital status, age, hospital, service, profession, contract type, shift and seniority in the profession.

Key words: Aggression. Workplace violence. Occupational health., Hospital nursing service. 


\section{INTRODUCCIÓN}

El despliegue de comportamientos violentos puede suponer un importante riesgo para la salud y seguridad de las trabajadores ${ }^{1}$. La Organización Mundial de la Salud (OMS) junto al Consejo Internacional de Enfermeras (CIE), la Organización Internacional del Trabajo (OIT) y la Organización Internacional de Servicios Públicos (ISP), definen la violencia laboral como "incidentes en los que el personal sufre abusos, amenazas o ataques en circunstancias relacionadas con su trabajo -incluidos los trayectos de ida y vuelta al mismo- que pongan en peligro, implícita o explícitamente, su seguridad, su bienestar o su salud"2. Según la forma de manifestarse, esta violencia se clasifica en violencia psicológica y violencia física. Se entiende por violencia física "el empleo de la fuerza física contra una persona o grupo, que produce daños físicos, sexuales o psicológicos, incluyendo las palizas, patadas, bofetadas, puñaladas, tiros, empujones, mordiscos y pellizcos". Y por violencia psicológica, "el uso deliberado del poder o amenazas al recurso de la fuerza física, contra una persona o grupo, que pueden dañar su desarrollo físico, mental, espiritual, moral o social. Comprende el abuso verbal, la intimidación, el atropello, el acoso y las amenazas" ${ }^{2}$. Es importante diferenciar los tipos de violencia, pues casi todos los profesionales están expuestos a insultos, pero la distribución no es igual en cuanto a agresiones físicas y tampoco en cuanto al comportamiento amenazante ${ }^{3}$.

El riesgo de violencia presenta una especial importancia en el sector servicios, ya que los trabajadores tienen una mayor probabilidad de sufrir agresiones o actos de violencia por parte de los usuarios y clientes, debido al estrecho contacto que mantienen con ellos ${ }^{2}$. Dentro de este sector, el sanitario ocupa un lugar destacado, existiendo estudios que dicen que más de la mitad de los trabajadores han experimentado al menos un incidente de violencia física o psicológica en el último año ${ }^{4}$.

Es difícil dar una cifra de incidencia/prevalencia de actos violentos en el ámbito sanitario, existiendo una gran variabilidad de datos debido a las diferentes metodologías empleadas y a la diversidad de instrumentos y criterios de evaluación utilizados en los estudios ${ }^{5-11}$. Así por ejemplo, mientras que Franz y colaboradores hablan de que el $70,7 \%$ de los trabajadores de una muestra alemana había sufrido una agresión física en los últimos 12 meses $^{9}$, otros trabajos, como el de Saarela e Isotalus en Finlandia, encuentran que uno de cada diez trabajadores del ámbito de la de salud había experimentado un incidente violento relacionado con el trabajo en el último año ${ }^{12}$.

Sin embargo existe consenso a la hora de indicar que el personal de enfermería es uno de los grupos profesionales con mayor incidencia de agresiones dentro del sector sanitario ${ }^{8,9,11,13}$. Sibbald, en un estudio realizado en la Columbia Británica, dice que en este colectivo la incidencia de violencia es casi cuatro veces la de cualquier otra profesión ${ }^{14}$ y Arnetz $\mathrm{y}$ colaboradores detectaron que un tercio de los profesionales de enfermería habían sufrido violencia en algún momento de su carrera ${ }^{15}$.

La importancia de este riesgo no sólo radica en la frecuencia con la que se presenta, sino también en la gravedad de las posibles consecuencias que pueden derivarse de él. Estas consecuencias pueden ser físicas, psicológicas y/o económicas para el trabajador, por ejemplo lesiones, burnout, cuadros psicopatológicos reactivos o abuso de sustancias ${ }^{7-9,16,17}$, pero también económicas para la empresa por 
el absentismo o perdida trabajadores capacitados $8,9,17$.

En España, desde los Sindicatos Profesionales como la Confederación Estatal de Sindicatos Médicos $(\mathrm{CESM})^{18}$, las Administraciones Sanitarias de diversas Comunidades Autónomas como Murcia $^{19}$ o Valencia ${ }^{20}$ y asociaciones como la Asociación Nacional para la Seguridad Integral en Centros Hospitalarios (AN$\mathrm{SICH})^{21}$, han desarrollado protocolos de prevención y declaración de estas agresiones, pero debemos tener en cuenta que, "muchos actos violentos no son registrados por sus víctimas por el miedo a disgustar al supervisor o director, por la presión del grupo de trabajo, por el miedo a perder el trabajo, por la excesiva burocracia que conlleva un registro o simplemente porque el personal de enfermería ha llegado a estar "entumecido" por la frecuencia de agresiones e insultos" 22 , lo que puede llevar a una infravaloración del riesgo. Este aspecto, junto al hecho de que estos programas permiten la declaración de incidentes y accidentes pero no la detección temprana de situaciones de riesgo de violencia, como aconsejan NTP $891^{23}$ y NTP $892^{24}$ hacen que por sí solos resulten insuficientes para valorar este riesgo.

Revisando los principales métodos utilizados para la evaluación de riesgos psicosociales en el ámbito laboral (Método $\mathrm{AIP}^{25}$ e ISTAS ${ }^{26}$ ), comprobamos que aunque detectan aspectos que pueden influir en la aparición de situaciones hostiles ${ }^{2,8,10}$, como una mala definición de las funciones del personal sanitario o la sobrecarga de trabajo, no permiten la identificación de situaciones o servicios con mayor riesgo. Respecto a los instrumentos específicos de valoración de conductas hostiles más habituales, como el LIPT-60 ${ }^{27}$, NAQ ${ }^{28}$ o IVAPT- PAN$\mathrm{DO}^{29}$, cabe señalar que valoran conduc- tas violentas por parte de compañeros o superiores pero no de los usuarios.

El objetivo de este estudio es conocer la frecuencia de aparición en el último año de diferentes manifestaciones hostiles de los usuarios hacia los profesionales de enfermería en los hospitales dependientes del Servicio Murciano de Salud (SMS), para valorar el clima de violencia laboral de baja intensidad y detectar aquellas características sociodemográficas y laborales de los trabajadores asociadas a una mayor exposición a este tipo de conductas.

\section{SUJETOS Y MÉTODOS}

Se trata de un estudio descriptivo transversal a través de un cuestionario autoaplicado, individual y anónimo, 1levado a cabo con el personal de enfermería de todos los hospitales públicos de la Región de Murcia durante el año 2010. Ese año el Servicio Murciano de Salud disponía de 11 hospitales con una plantilla total de 19.550 de los cuales 6.103 eran personal de enfermería (enfermeros y auxiliares de enfermería) en atención especializada. El muestreo se estratificó en función del tamaño de las unidades y de la plantilla de los hospitales, obteniendo una muestra total de 1.489 sanitarios que cumplimentaron el protocolo de investigación. Teniendo en cuenta los datos del Servicio Murciano de Salud, la muestra supone el 28,98\% del total del personal de enfermería y el 18,96\% del total de los auxiliares de enfermería hospitalarios de la Región. La tasa de respuesta global fue del 70,48\%. De esta muestra se deriva un nivel de confianza del 99\% y un error muestral del 1,75\%.

La muestra estuvo compuesta por 1.489 profesionales de enfermería con un rango de edad que osciló entre 20 y 67 años, siendo la edad media de 42,09. La 
mayoría eran mujeres ( $82,7 \%$ vs. $16,4 \%)$ y el $67,8 \%$ estaba casado o con pareja de hecho.

Para obtener la muestra el equipo de investigación se puso en contacto con las direcciones de enfermería de los centros a quien se informó detalladamente del estudio. Una vez que los hospitales aceptaron la participación en la investigación, se convocaron reuniones en cada uno de ellos a las que asistieron todos los supervisores de las diferentes unidades del hospital para informarles sobre el estudio y repartirles el protocolo de investigación. Se dieron instrucciones para que cada supervisor entregara, de forma aleatoria, al 30\% del personal que tenía a su cargo un protocolo para ser rellenado individualmente. El trabajador recibía la encuesta en un sobre donde debía guardarla tras contestarla y entregarlo sin identificar y cerrado a su superior, el cual se encargaba de su devolución a los entrevistadores, siendo éstos abiertos por miembros del equipo de investigación, que les asignaron un código a efectos del análisis de datos.

En cada hospital se asignó un número de colaboradores según el tamaño de la muestra. Ellos visitaban el hospital dos veces por semana para comprobar el proceso de recogida de protocolos junto con las personas propuestas por el hospital (normalmente responsables de docencia e investigación).

El muestreo se realizó durante los tres primeros meses del año 2010, dando de plazo para la recogida de los protocolos un tiempo máximo de dos semanas desde la entrega de los cuestionarios. Los que no se recogieron en ese plazo se dieron por perdidos.

El instrumento de medida utilizado fue la escala Escla de Conductas Hosti-
les-Usuarios (ECOH-U) ${ }^{30}$ cuyo objetivo es medir manifestaciones hostiles verbales y físicas de baja intensidad procedentes de los usuarios y percibidas por el trabajador como violentas. Para valorar la frecuencia se estableció una escala tipo Likert con 6 opciones de respuesta que valora la frecuencia de aparición de cada situación $(1=$ nunca en el último año, $2=$ anualmente, $3=$ trimestralmente, $4=$ mensualmente, $5=$ semanalmente, $6=$ diariamente). El cuestionario está configurado por 10 ítems con un alpha total de 0,839 , explicando el $57,36 \%$ de la varianza, y se divide en dos factores: Violencia verbal (alpha $=0,85$ explicando el $36,39 \%$ de la varianza con una carga factorial de 0,636-0,756) con 7 ítems y Violencia física de 3 items (alpha $=0,74$ explicando el 20.96 de la varianza y una carga factorial de 0,713-0,782) de 3 ítems ${ }^{30}$.

Para el análisis bivariado, junto a este cuestionario se registraron una serie de variables sociodemográficas (sexo, edad y estado civil) y laborales del trabajador tales como: hospital, tipo de servicio, profesión, antigüedad en el puesto (agrupado para el análisis estadístico: $<1,1-5$, $6-10,11-15$ o >15) y en la profesión (agrupado para estudio: $<5,6-10,11-20$ o $>20$ ), tipo de contrato, turno, si realiza formación continuada, si se realizan otros trabajos u horas extra. Además se añadieron dos preguntas para valorar la prevalencia de agresiones: ¿Ha sufrido una agresión física en los últimos 6 meses? ¿Ha visto agredir a un compañero en los últimos 6 meses?

El análisis estadístico se inició con la distribución de la muestra, presentando los porcentajes de respuesta según variables sociodemográficas y laborales. Posteriormente se recogió el número y porcentaje de trabajadores que refirió cada una de las manifestaciones de violencia 
que componen el cuestionario, según la frecuencia de exposición. Para el análisis de las puntuación medias obtenidas en la Ecoh-U según las distintas variables se utilizó la prueba t de Student para medias obtenidas en variables dicotómicas, realizando previamente un análisis de varianzas por medio de la correlación de Pearson, y la prueba de ANOVA para variables multirrespuesta, utilizando, en estos casos, la prueba post hoc de Tukey para el establecimiento de los diferentes grupos.

El análisis estadístico se efectuó con el programa SPSS en su versión 15.0, y el nivel de significación asumido en todos los contrastes fue de 0,05 .

\section{RESULTADOS}

En la tabla 1 puede verse que el $62,1 \%$ de la muestra eran profesionales de enfermería y el $36,7 \%$ eran auxiliares de enfermería. Los profesionales tenían una media de antigüedad en el puesto actual de 7 años y 5 meses y la media de la antigüedad en la profesión como enfermero/a o auxiliar de enfermería era de 15 años y 4 meses . El 59,4\% de la muestra tenía contrato indefinido frente a un $33 \%$ de contratos temporales. El 50,5\% estaban en turno rotatorio, un $29,9 \%$ en turno de mañana, un $2,5 \%$ turno de noche y un $16 \%$ tenía otro tipo de turno. La mayoría no hacían horas extras al mes o hacían menos de $10(74,5 \%)$ y un $6,2 \%$ realizaba otra actividad laboral. Principalmente trabajaban en unidades de hospitalización de adultos $(33,2 \%)$.

El 22,8\% de los trabajadores sufrieron semanal o diariamente al menos una de las manifestaciones de violencia verbal recogida en la escala, alcanzando el $71 \%$ si consideramos una frecuencia al menos anual. Respecto a la violencia física, el $1,2 \%$ de los trabajadores sufrieron semanal o diariamente al menos una de las
Tabla 1

Características sociodemográficas y laborales de las personas de la muestra

\begin{tabular}{|c|c|c|}
\hline Variables & $\mathrm{n}$ & $\%$ \\
\hline Varón & 244 & 16,4 \\
\hline Mujer & 1231 & 82,7 \\
\hline Estado civil & 386 & 25,9 \\
\hline Pareja de hecho y casados & 1009 & 67,8 \\
\hline Divorciados, separados y viudos & 85 & 5,7 \\
\hline Edad & 201 & 13,5 \\
\hline $30-50$ & 864 & 58 \\
\hline$>50$ & 327 & 22 \\
\hline Hospital 1 & 432 & 29 \\
\hline Hospital 2 & 295 & 19,8 \\
\hline Hospital 3 & 141 & 9,5 \\
\hline Hospital 4 & 46 & 3,1 \\
\hline Hospital 5 & 122 & 8,2 \\
\hline Hospital 6 & 94 & 6,3 \\
\hline Hospital 7 & 132 & 8,9 \\
\hline Hospital 8 & 42 & 2,8 \\
\hline Hospital 9 & 63 & 4,2 \\
\hline Hospital 10 & 45 & 3 \\
\hline Hospital 11 & 77 & 5,2 \\
\hline \begin{tabular}{|l|} 
Servicios \\
Principalmente quirúrgicos
\end{tabular} & 189 & 12,7 \\
\hline Principalmente clínicos & 175 & 11,8 \\
\hline Otros servicios hospitalización & 130 & 8,7 \\
\hline Quirófanos y Reanimación & 152 & 10,2 \\
\hline Urgencias & 137 & 9,02 \\
\hline Uci & 71 & 4,8 \\
\hline Consultas Externas & 114 & 7,7 \\
\hline Psiquiatría & 77 & 5,2 \\
\hline Materno-Infantil & 176 & 11,8 \\
\hline Otros & 76 & 5,1 \\
\hline Enfermería & 925 & 62,7 \\
\hline Auxiliar de enfermería & 546 & 36,7 \\
\hline Antigüedad en la profesión $\quad<5$ & 141 & 9,5 \\
\hline $06-11$ & 388 & 26,1 \\
\hline $11-20$ & 487 & 32,7 \\
\hline$>20$ & 384 & 25,8 \\
\hline
\end{tabular}


Tabla 1

Características sociodemográficas y laborales de las personas de la muestra (continuación)

\begin{tabular}{|c|c|c|}
\hline Variable & $\mathrm{n}$ & $\%$ \\
\hline Antigüedad en el puesto & 202 & 13,6 \\
\hline $1-5$ & 577 & 38,8 \\
\hline $6-10$ & 294 & 19,7 \\
\hline $11-15$ & 151 & 10,1 \\
\hline$>15$ & 203 & 13,6 \\
\hline Fijo (indefinido) & 884 & 59,4 \\
\hline Temporal & 492 & 33 \\
\hline Fijo mañana & 445 & 29,9 \\
\hline Fijo noche & 37 & 2,5 \\
\hline Rotatorio & 752 & 50,5 \\
\hline Otros (Turno mañana/tarde) & 238 & 16 \\
\hline Horas extra & 1.110 & 74,5 \\
\hline $10-30$ horas & 217 & 14,6 \\
\hline$>30$ horas & 57 & 3,8 \\
\hline Formación continuada & 1.011 & 67,9 \\
\hline No & 427 & 28,7 \\
\hline Otra actividad laboral & 93 & 6,2 \\
\hline No & 1.377 & 92,5 \\
\hline
\end{tabular}

manifestaciones descritas, refiriendo una frecuencia al menos anual el 19,9\% de la muestra.

Los trabajadores refirieron que las conductas de violencia más frecuentes (al menos mensual) fueron en primer lugar, los enfados por la demora asistencial en un $21,8 \%$ de los encuestados y en segundo, las malas caras y las miradas de desprecio referidas por un $9,5 \%$ de los trabajadores. Las manifestaciones menos habituales fueron las destrucciones de puertas y cristales y los empujones y zarandeos, ya que el 92,7\% de trabajadores para el primero y el $90,1 \%$ para el segundo, respondieron "nunca en el último año" (tabla 2).

Teniendo en cuenta las variables sociodemográficas (tabla 3 ), los trabaja- dores solteros obtuvieron una puntuación total media significativamente mayor que las personas viudas/separadas/ divorciadas (17,54 vs 14,19; $\mathrm{p}<0,001)$. Ocurrió lo mismo con la puntuación por los trabajadores menores de 30 años frente a los mayores de 50 años $(17,94$ vs 14,$58 ; p<0,001)$. No se observaron diferencias significativas en la puntuación media de la escala según sexo.

En la tabla 4 se muestran las puntuaciones obtenidas según las variables laborales. Por hospital, las pruebas post hoc (Tukey) revelaron que los trabajadores del hospital psiquiátrico de la región, tuvieron una puntuación mayor, estadísticamente significativa, que el personal del resto de hospitales, en la puntuación total de la escala $(19,69 ; \mathrm{p}<0,001)$ y en la puntuación de violencia física de $(5,33$; $\mathrm{p}<0,01)$. Por servicios, los trabajadores de quirófano y reanimación son los que menor puntuación obtuvieron $(12,59) \mathrm{y}$ los de Urgencias los que mayor $(20,60)$, existiendo diferencias de puntuación estadísticamnete significativas tanto en violencia física como en verbal. Con respecto a la profesión, las enfermeras tuvieron una puntuación significativamente más alta en violencia verbal que las auxiliares de enfermería, (12,93 vs $11,67 ; \mathrm{p}<0,001)$, así como en la puntuación total $(16,35$ vs 15,$14 ; \mathrm{p}<0,01)$. Según el tipo de contrato, las puntuaciones en violencia total fueron significativamente superiores en los temporales respecto a los fijos (16,38 vs 15,62 ; $\mathrm{p}<0,05)$. Por turno, encontramos una puntuación media de violencia física $(4,00)$ significativamente mayor $(\mathrm{p}<0,001)$ para el turno de noche. Las personas que más horas extras hacían obtuvieron puntuaciones medias significativamente superiores en violencia física $(3,74 ; \mathrm{p}<0,05)$ frente a aquellas que no las hacían, mientras que los que reali- 


\section{Tabla 2}

\section{Frecuencia de exposición a conductas violentas}

\begin{tabular}{|c|c|c|c|c|c|c|}
\hline & $\begin{array}{c}\text { Nunca } \\
\mathrm{n}(\%)\end{array}$ & $\begin{array}{l}\text { Anual } \\
\mathrm{n}(\%)\end{array}$ & $\begin{array}{c}\text { Trimestral } \\
\mathrm{n}(\%)\end{array}$ & $\begin{array}{c}\text { Mensual } \\
\mathrm{n}(\%)\end{array}$ & $\begin{array}{c}\text { Semanal } \\
\mathrm{n}(\%)\end{array}$ & $\begin{array}{l}\text { Diario } \\
\mathrm{n}(\%)\end{array}$ \\
\hline \multicolumn{7}{|l|}{ Violencia verbal } \\
\hline Enfados por demora asistencial & $648(44,8)$ & $341(23,2)$ & $150(10,2)$ & $111(7,6)$ & $100(6,8)$ & $109(7,4)$ \\
\hline Malas caras o miradas de desprecio & $858(58,4)$ & $368(25,1)$ & $194(7,1)$ & $64(4,4)$ & $46(3,1)$ & $29(2)$ \\
\hline Cuestionar las decisiones & $890(60,9)$ & $327(22,4)$ & $87(6)$ & $69(4,7)$ & $60(4,1)$ & $28(1,9)$ \\
\hline Bromas irónicas & $903(61,6)$ & $345(23,5)$ & $91(6)$ & $59(4)$ & $44(3)$ & $25(1,7)$ \\
\hline Enfados por la falta de información & $905(61,6)$ & $322(21,9)$ & $82(5,6)$ & $64(4,4)$ & $55(3,7)$ & $41(2,8)$ \\
\hline Enfados exagerados por minucia & $907(61,7)$ & $283(19,3)$ & $103(7)$ & $72(4,9)$ & $64(4,4)$ & $41(2,8)$ \\
\hline Acusaciones injustificadas & $1032(70,6)$ & $284(19,4)$ & $65(4,4)$ & $33(2,3)$ & $29(2)$ & $18(1,2)$ \\
\hline \multicolumn{7}{|l|}{ Violencia física } \\
\hline Sujeciones hostiles & $1279(87,1)$ & $143(9,7)$ & $29(2)$ & $12(0,8)$ & $4(0,3)$ & $2(0,1)$ \\
\hline Empujones, zarandeos... & $1324(90,1)$ & $101(6,9)$ & $28(1,9)$ & $11(0,7)$ & $4(0,3)$ & $2(0,1)$ \\
\hline Destrucción de puertas, cristales ... & $1362(92,7)$ & $77(5,2)$ & $15(1)$ & $6(0,4)$ & $6(0,4)$ & $4(0,3)$ \\
\hline
\end{tabular}

Tabla 3

Exposición a violencia según variables sociodemográficas y laborales

\begin{tabular}{|c|c|c|c|c|c|c|c|c|c|}
\hline & \multicolumn{3}{|c|}{ Agresión fisica } & \multicolumn{3}{|c|}{ Agresión verbal } & \multicolumn{3}{|c|}{ Agresión total } \\
\hline & Media & DT & $\mathrm{p}$ & Media & DT & $\mathrm{p}$ & Media & DT & $\mathrm{p}$ \\
\hline \multicolumn{10}{|l|}{ Sexo } \\
\hline Varón & 3,57 & 1,52 & & 12,7 & 6,27 & \multirow{3}{*}{$\mathrm{NS}^{\mathrm{a}}$} & 16,27 & 7,03 & \multirow{2}{*}{$\mathrm{NS}^{\mathrm{a}}$} \\
\hline Mujer & 3,42 & 1,22 & 105 & 12,44 & 6,24 & & 15,85 & 6,83 & \\
\hline \multicolumn{9}{|l|}{ Estado civil } & \\
\hline Solteros & 3,57 & 1,42 & \multirow{3}{*}{$<0,05^{b}$} & 13,97 & 6,65 & \multirow{3}{*}{$<0,001^{\mathrm{b}}$} & 17,54 & 7,3 & \multirow{3}{*}{$<0,001^{\mathrm{b}}$} \\
\hline Pareja de hecho y casados & 3,41 & 1,25 & & 12,02 & 6,09 & & 15,42 & 6,71 & \\
\hline Divorciados, separados y viudos & 3,24 & 0,62 & & 10,94 & 5,03 & & 14,19 & 5,19 & \\
\hline \multicolumn{10}{|l|}{ Edad } \\
\hline$<30$ & 3,5 & 1,16 & \multirow{3}{*}{$<0,05^{b}$} & 14,44 & 6,54 & \multirow{3}{*}{$<0,001^{b}$} & 17,94 & 6,99 & \multirow{3}{*}{$<0,001^{b}$} \\
\hline $30-50$ & 3,5 & 1,37 & & 12,41 & 6,24 & & 15,91 & 6,98 & \\
\hline$>50$ & 3,27 & 1,08 & & 11,31 & 5,62 & & 14,58 & 6 & \\
\hline
\end{tabular}

a Prueba t. ${ }^{\mathrm{b}} \mathrm{ANOVA}$

zaban formación continuada tuvieron puntuaciones mayores en la violencia verbal y total $(12,75$ y 16,22 respectivamente; $p<0,05)$. Según la antigüedad en la profesión, se observaron puntuaciones significativamente superiores en los trabajadores que llevaban trabajando entre
6-10 años $(17,20 ; p<0,001)$ frente a los trabajadores con más de 20 , cuya puntuación media fue 14,96.

E1 3\% de los trabajadores sufrieron agresiones físicas en los últimos 6 meses y el 7,5\% refirieron haber visto agredir a 


\section{Tabla 4}

Exposición a violencia según variables laborales

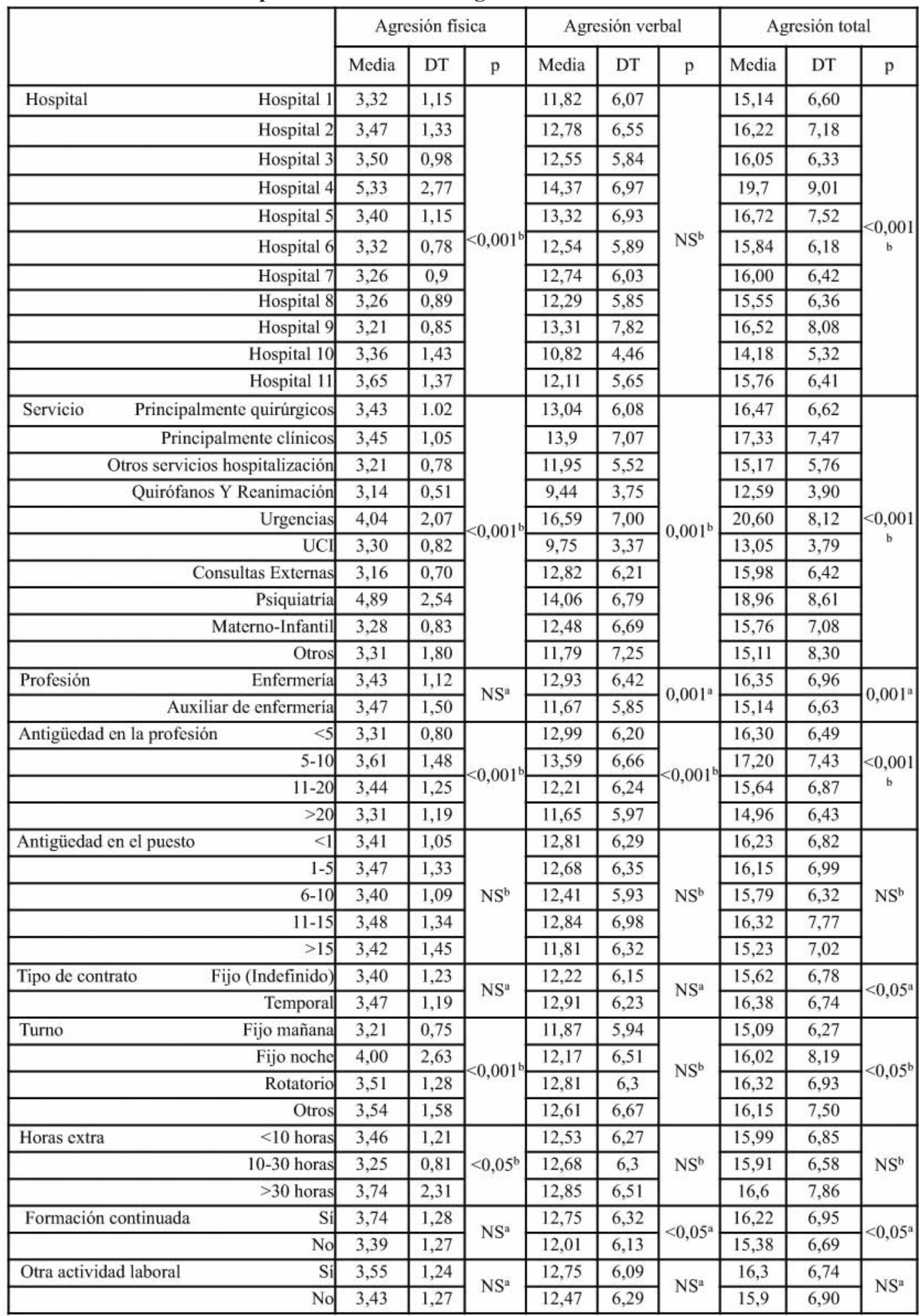

${ }^{\mathrm{a}}$ Prueba t. ${ }^{\mathrm{b}} \mathrm{ANOVA}$ 


\section{Tabla 5}

Puntuaciones obtenidas según ocurrencia de agresiones en los últimos 6 meses

\begin{tabular}{|c|c|c|c|c|c|c|c|c|c|}
\hline & \multicolumn{3}{|c|}{ Agresión física } & \multicolumn{3}{|c|}{ Agresión verbal } & \multicolumn{3}{|c|}{ Agresión total } \\
\hline & Media & DT & $\mathrm{p}$ & Media & DT & $\mathrm{p}$ & Media & DT & $\mathrm{p}$ \\
\hline Ha sufrido agresiones fisicas $\mathrm{Si}$ & 5,61 & 2,88 & \multirow{2}{*}{$<0,001^{\mathrm{a}}$} & 18,07 & 7,78 & \multirow{2}{*}{$<0,001^{\mathrm{a}}$} & 23,7 & 9,68 & \multirow{2}{*}{$<0,001^{\mathrm{a}}$} \\
\hline No & 3,36 & 1,07 & & 12,31 & 6,09 & & 15,66 & 6,54 & \\
\hline \multirow[t]{2}{*}{ Ha visto agresiones fisicas } & 4,74 & 2,55 & \multirow{2}{*}{$<0,001^{\mathrm{a}}$} & 16,46 & 8,41 & \multirow{2}{*}{$<0,001^{\mathrm{a}}$} & 21,17 & 9,89 & \multirow{2}{*}{$<0,001^{\mathrm{a}}$} \\
\hline & 3,31 & 0,98 & & 12,14 & 5,89 & & 15,45 & 6,29 & \\
\hline
\end{tabular}

un compañero. De forma general, la media de puntuación en la escala obtenida por estas personas fue significativamente superior $(p<0,001)$ respecto aquellos cuya respuesta había sido negativa (tabla 5).

\section{DISCUSIÓN}

En general podemos decir que en este estudio las cifras de agresiones detectadas entre el personal de enfermería son inferiores a las de otras investigaciones, si consideramos que sólo el 3\% respondió afirmativamente ante la pregunta si han sufrido agresiones físicas en los últimos 6 meses, frente a otros estudios donde hablan de un $14,4 \%$ de agresiones físicas en los últimos 5 turnos $^{8}$ o un $44,8 \%$ de agredidos físicamente al mes, subiendo al 70,7\% cuando hablan del último año ${ }^{9}$, aunque debemos indicar que no se ha utilizado la misma metodología. Coincidimos con estos estu$\operatorname{dios}^{7-9}$ en afirmar que son más frecuentes las manifestaciones verbales de violencia que las físicas.

Con este trabajo hemos puesto de manifiesto que aunque el personal de enfermería ocupe un lugar destacado entre las profesiones más expuestas a violencia por parte de los usuarios ${ }^{4}$, la distribución de este riesgo no es homogénea, existiendo diferencias tanto en la frecuencia de exposición (violencia total) como en el tipo de mani- festación (violencia física/violencia verbal) según variables laborales y personales del trabajador, que pueden orientar la intervención dirigida a la prevención de estos hechos.

Igual que en los estudios de Roche ${ }^{8}$ o Shields ${ }^{13}$ identificamos como posibles factores de riesgo para la exposición a violencia de usuarios, la baja experiencia en la profesión y tener una menor edad. En este sentido, Whittington ${ }^{31}$ refiere en su estudio del 2002 que el personal con más de 15 años de antigüedad muestran una mayor tolerancia a los comportamientos violentos de los pacientes, posiblemente por el desarrollo de una "sabiduría profesional" y un aumento de la confianza en el trato con los pacientes agresivos. En consecuencia consideramos que la formación en el manejo adecuado de estas situaciones conflictivas y/o el trato con el paciente, podrían llevar a una reducción de las agresiones ${ }^{2,8,32}$. Asociada a este punto, aparece también como factor de riesgo el estado civil. Esto puede ser debido a que se encuentre anidado con la edad.

Respecto a la consideración del servicio u hospital donde trabaja el profesional como factor de riesgo, destacamos, como en otros estudios ${ }^{7,12}$, los servicios de urgencias y psiquiatría por su mayor prevalencia. La Occupational Safety and 
Health Administration (OSHA) $)^{32}$ identificó como factores de riesgo que promueven la violencia en los hospitales la organización del trabajo, el diseño ambiental, las conductas del personal y el tipo de pacientes. Parece que este último factor podría ser fundamental para explicar la mayor puntuación en unidades de psiquiatría, pero también en urgencias, pues estudios como el de Crilly ${ }^{10}$, realizado en personal de enfermería de urgencias, identifica como factores de riesgo de violencia de los pacientes el consumo de alcohol o drogas y comportamientos asociados con una enfermedad mental. Debemos tener en cuenta que la violencia ejercida por enfermos mentales fuera del hospital es escasa, equiparable a la de la población general, salvo cuando sufren una descompensación de su enfermedad, pero es en esta situación cuando suelen ser atendidos en los servicios de urgencias y en las unidades psiquiátricas, donde además son asistidos, en ocasiones, a petición de terceros, siendo un pequeño grupo de pacientes los responsables de un porcentaje elevado de episodios agresi$\operatorname{vos}^{33}$. Aunque este factor no sería modificable,indica la necesidad de una formación específica para los trabajadores de estos servicios, pues como señala Mill y Rose $^{34}$ cuando los niveles de comportamientos desafiantes no se pueden reducir, las intervenciones deben centrarse en el personal, con la puesta en marcha de programas que le ayuden a hacer frente a estas situaciones.

En relación a los turnos, Gacki-Smith ${ }^{35}$ nos habla del turno de noche y Shields ${ }^{13}$ del rotatorio como factores de riesgo. En nuestro estudio hemos encontrado diferencias estadísticamente significativas en relación a la violencia física pero no en las manifestaciones verbales. Consideramos que el aumento de este tipo de manifestaciones se puede deber a la menor presencia de personal en los servicios en los turnos de tarde y noche, así como por el tipo de paciente atendido, por lo que recomendamos el aumento de personal de seguridad en estos turnos.
En otros trabajos en los que se ha evaluado la exposición de violencia a través de la medición de agresiones, se habla de una menor declaración de agresiones del sexo femenino ${ }^{35,13}$, sin embargo, en nuestro estudio no hemos obtenido diferencias estadísticamente significativas según el sexo del trabajador. Esta diferencia puede ser debida al uso de un método de recogida de datos diferente (cuantificación de sucesos declarados vs recogida de datos a través de una encuesta).

La aplicación de esta escala para evaluar el riesgo ha permitido la detección temprana de los grupos con mayor exposición a climas de violencia laboral de baja intensidad ${ }^{23,24}$, lo que permitiría la priorización y selección de las medidas preventivas colectivas e individuales más adecuadas dirigidas a reducir las afectaciones psicológicas asociadas a la exposición continuada a situaciones de violencia $^{7-9,16,17}$. Igualmente, se ha puesto de manifiesto que los trabajadores que han sufrido agresiones físicas o han visto agredir físicamente a compañeros en los últimos 6 meses han estado más expuestos a violencia tanto físicas como verbales de baja intensidad en el último año, por lo que consideramos que interviniendo sobre los factores favorecedores de un clima de violencia podemos reducir la frecuencia de lesiones debidas a agresiones. Por estas dos razones, proponemos la inclusión de la valoración del riesgo de violencia de los usuarios en la evaluación de riesgos psicosociales de todos los puestos de trabajo con atención al público.

Indicar que los profesionales de enfermería no son los únicos de la sanidad que están expuestos a la violencia de los usuarios. Una crítica habitual es que la mayoría de los estudios se han realizado con muestras formadas principalmente por estos profesionales, pero Gascon ${ }^{36}$ en 
su estudio sobre agresiones analizó las categoría profesional como factor de riesgo detectando una asociación directa entre el nivel de responsabilidad y la exposición a violencia no física, de tal forma que los médicos y directivos eran el personal más expuesto. Por ello, para próximas investigaciones, sugerimos ampliar el estudio al resto de profesionales de la salud.

Por último señalar que, como en todo estudio basado en cuestionarios autoaplicados, podríamos haber cometido un sesgo de autoselección, pero se ha aleatorizado la selección de los participantes y se ha asegurado su anonimato para minimizar este problema.

\section{AGRADECIMIENTOS}

Queremos manifestar nuestro agradecimiento a todos los trabajadores que han contestado al cuestionario de la investigación, por su tiempo y dedicación, a las direcciones de los centros donde se ha llevado a cabo el estudio, por su colaboración, y a los estudiantes de postgrado que han participado en la recogida de datos, por su trabajo.

\section{BIBLIOGRAFÍA}

1. INSHT. NTP 489: Violencia en el lugar de trabajo. Madrid: Instituto Nacional de Seguridad e Higiene en el Trabajo. Ministerio de Trabajo e Inmigración; 1998 .

2. OIT/CIE/OMS/ISP. Directrices marco para afrontar la violencia laboral en el sector de la salud. Ginebra: Organización Internacional del Trabajo; 2002.

3. Martínez Jarreta B, Gascón S, Santed MA, Goicoechea J. Análisis médico-legal de las agresiones a profesionales sanitarios: aproximación a una realidad silenciosa y a sus consecuencias para la salud. Med Clin (Barc). 2007;128(8):307-10.

4. Di Martino V. Workplace violence in the health sector. Country case studies Brazil, Bulgaria, Lebanon, Portugal, South Africa, Thailand and an additional Australian study. Ginebra: Organización Internacional del Trabajo; 2002.
5. Ayranci U. Violence Toward Health Care WorKers in Emergency Departments in West Turkey. J Em erg M ed. $2005 ; 28(3): 361-5$. http://dx.doi.org/10.1016/ j.jemermed.2004.11.018.

6.Sanuzun F, Keradokovan A. Violence Towards Nursing Staff in Emergency Deparment in one Turkish City. Int Nurs Rev. 2005;52(2): 154-60. http://dx.doi.org/10.1111/j.1466-7657.2005.00420.x

7. Winstanley S, Whittington R. Aggression towards health care staff in a UK general hospital: variation among professions and departments. J Clin Nurs. 2004;13(1):3-10. http://dx.doi.org/10.1111/j.13652702.2004.00807

8. Roche M, Diers D, Duffield C, Catling-Paull C. Violence Toward Nurses, the Work Environment, and $\mathrm{Patient} \mathrm{Outcomes.} \mathrm{J} \mathrm{Nurs} \mathrm{Scholarsh.}$ 2010;42(1):13-22. http://dx.doi.org/10.1111/j.15475069.2009.01321.x.

9. Franz S, Zeh A, Schablon A, Kuhnert S, Nienhaus A. Aggression and violence against health care workers in Germany- a cross sectional retrospective surve. BMC Health Serv Res [edición electrónica]. 2010; 10. Disponible en: http://www.biomedcentral.com/14726963/10/51. http://dx.doi.org/10.1186/1472-6963-1051.

10. Crilly J, Chaboyer W, Creedy D. Violence towards emergency department: nurses by patients. Accid Emerg Nurs. $2004 ; 12(2): 67-73$. http://dx.doi.org/10.1016/ j.aaen.2003.11.003.

11. Kling RN, Yassi AN, Smailes E, Lovato CY, Koehoorn M. Characterizing violence in health care in British Columbia. J Adv Nurs. 2009;65(8):1655-63. http://dx.doi.org/ 10.1111/j.1365-2648.2009.05020.x.

12. Saarela KL, Isotalus N. Workplace Violence in Finland: High-risk groups and preventive strategies. Am. J. Ind. Med. 1999;36 Sup 1 1:80-1. ht tp://dx.doi.org/10.1002/( S I C I) 1097 0274(199909)36:1+<80::AID-AJIM29>3.0.CO;2-U.

13. Shields M, Wilkins K. Factors related to on-the-job abuse of nurses by patients. Health Rep. 2009;20(2):114.

14. Sibbald B. Physician, protect thyself. CMAJ. 1998;159(8):983-5.

15. Arnetz JE, Arnetz BB, Petterson IL. Violence in the nursing profession: occupational and lifestyle risk factors in Swedish nurses. Work Stress. 1996;10(2):11927. http://dx.doi.org/10.1080/02678379608256791. 
16. Estryn-Behar M, Van del Heijden B, Camerino D, Fry C, Le Nezet O, Conway PM et al. Violence risks in nursing-results from the European "NEXT" Study. Occup Med (Chic Ill). 2008;58(2):107-14. http://dx.doi.org/10.1093/occmed/kqm142.

17. Quine L. Workplace bullying in NHS community trust: staff questionnaire survey. $\mathrm{Br}$ Med J. 1999 ; $\quad 318: 228-32$ http://dx.doi.org/10.1136/bmj.318.7178.228.

18. CESM. Documento Marco para la prevención de la violencia hacia los facultativos. Madrid: Confederación Estatal de Sindicatos Médicos; 2007.

19. Servicio Murciano de Salud. Plan de prevención de las agresiones a los profesionales de la sanidad de la Región de Murcia. Murcia: Servicio Murciano de Salud. Dirección General de Recursos Humanos; 2005 .

20. Generalitat Valenciana. Plan Integral de Prevención y Atención de las Agresiones a los Trabajadores del Sector Sanitario Público de la Generalitat. Valencia: Servicio de Prevención de Riesgos Laborales. Conselleria de Sanidad. Generalitat Valenciana; 2010 .

21. ANSICH. Manual de prevención ante las agresiones al personal sanitario. Asociación Nacional para la seguridad integral en centros hospitalarios. Barcelona: Alternativa Group; 2011.

22. Rippon TJ. Aggression and violence in health care professions. J Adv Nurs. 2000;31(2):452-60. ht t p : / / dx.doi.org/ $10.1046 /$ j. 1365 2648.2000.01284.x.

23. INSHT. NTP 891: Procedimiento de solución autónoma de los conflictos de violencia laboral (I). Madrid: Instituto Nacional de Seguridad e Higiene en el Trabajo. Ministerio de Trabajo e Inmigración; 2011.

24. INSHT. NTP 892: Procedimiento de solución autónoma de los conflictos de violencia laboral (II). Madrid: Instituto Nacional de Seguridad e Higiene en el Trabajo. Ministerio de Trabajo e Inmigración; 2011.

25. Generalitat de Catalunya .Manual del Método PSQ CAT21 COPSOQ (Versión 1.5) para la evaluación y prevención de los riesgos psicosociales en las empresas de menos de 25 trabajadores y trabajadoras. Barcelona: Generalitat de Catalunya. Departamento de Trabajo. Dirección General de Relaciones Laborales; 2010.
26.INSHT. NTP 443: Factores psicosociales: metodología de evaluación. Madrid: Instituto Nacional de Seguridad e Higiene en el Trabajo. Ministerio de Trabajo e Inmigración; 1997.

27. González JL, Rodríguez-Abuin MJ. Cuestionario de estrategias de acoso en el trabajo. El LIPT-60. Madrid: Editorial EOS; 2005.

28. Einarsen S, Hoel H, Notelaers G. Measuring exposure to bullying and harassment at work: Validity, factor structure and psychometric properties of the Negative Acts Questionnaire-Revised. Work Stress. 2009;23(1):24-44. http://dx.doi.org/10.1080/ 02678370902815673 .

29. Pando M, Aranda C, Preciado L, Franco SA, Salazar JG. Validez y confiabilidad del inventario de violencia y acoso psicológico en el trabajo (IVAPT-PANDO). Enseñanza e Investigación en Psicología. 2006;11(2):319-32.

30. Waschgler K, Ruiz JA, Llor B. Patients'Aggressive Behaviours towards Nurses: A Scale Development. J Adv Nurs. En prensa.

31. Wittington R. Attitudes toward patient aggression amongst mental health nurses in the 'zero tolerance' era: associations with burnout and length of experience. J Clin Nurs. 2002;11(6):819-25. http://dx.doi.org/10.1046/j.1365-2702.2002.00659.x

32. OSHA. Guidelines for Preventing Workplace Violence for Health Care and Social Service Workers. Washington: OSHA. U.S. Department of Labor; 2004.

33. Gascón J, Rojo JE, Salazar A, Tomás S. Guía Práctica de Manejo Terapéutico y Prevención en el Paciente Agitado. Master Line \& Prodigio, S.L. Madrid, 2004.

34. Mills S, Rose J. The relationship between challenging behaviour, burnout and cognitive variables in staff working with people who have intellectual disabilities. J Intellect Disabil Res. 2011;55(9):844-57. http://dx.doi.org/ 10.1111/j.1365-2788.2011.01438.x

35. Gacki-Smith J, Juarez AM, Boyett L, Homeyer C, Robinson L, MacLean SL. Violence Against Nurses Working in US Emergency Departments. J Nurs Adm. $20009 \% 39(7 / 8): 340-9$. http://dx.doi.org/10.1097/NNA.0b013e3181ae97d.

36. Gascon S, Martínez-Jarreta B, González-Andrade JF, Santed MA, Casalod Y, Rueda MA. Aggression Towards Health Care Workers in Spain:A Multi-facility Study to Evaluate the Distribution of a Growing Violence Among Professionals, Health Facilities and Departments. Int J Occup Environ Health. 2009;15(1):30-6. 
37. Tak S, Sweeney MH, Alterman T, Baron S, Calvert GM. Workplace Assaults on Nursing Assistants in US Nursing Homes: A Multilevel Analysis. Am J Public Health. 2010;100(10):1938-45. http://dx.doi.org/ 10.2105/AJPH.2009.185421.

38. Gates D, Ross C, McQueen L. Violence against Emergency Department Workers. J Emerg Med. 2006;31:331-7.

39. Jansen G, Dassen T, Jebbink G. Staff attitudes towards aggression in health care: a review of the literature. J Psychiatr Ment Health Nurs. 2004;12(3):3-13. http://dx.doi.org/ 10.1016/j.jemermed.2005.12.028. 